\title{
La universidad como ruptura en la trayectoria educativa: experiencias de transición de estudiantes egresados de Enseñanza Media Técnico Profesional que ingresan al Programa Académico de Bachillerato de la Universidad de Chile*
}

\author{
University as an educational trajectory rupture: transition experience of Professional \\ Technical High School graduates that enter to Universidad de Chile's Programa de \\ Bachillerato
}

\author{
Irina López Cárdenas ${ }^{a}$, Julio Mella Luna ${ }^{b}$, Gabriela Cáceres Valenzuela ${ }^{c}$ \\ a Programa Académico de Bachillerato, Universidad de Chile \\ Correo electrónico: irinalopezc @uchile.cl \\ b Programa Académico de Bachillerato, Universidad de Chile \\ Correo electrónico: jmella@uchile.cl \\ ${ }^{\mathrm{c}}$ Programa Académico de Bachillerato, Universidad de Chile \\ Correo electrónico: gabriela.caceres @ug.uchile.cl
}

\begin{abstract}
RESUMEN
El presente estudio explora la experiencia de transición a la universidad de estudiantes egresados de Enseñanza Media Técnico Profesional (EMTP) que ingresan al Programa Académico de Bachillerato de la Universidad de Chile. Mediante entrevistas semiestructuradas realizadas a 6 estudiantes que cursaban el primer año en el Programa el año 2017, se indagó su proceso de transición a la universidad. Los resultados dan cuenta de: i) que el ingreso a la universidad significa un Quiebre de Trayectoria del estudiante. Dada la posibilidad de ingresar a la universidad, el estudiante debe redefinir su proyecto de vida y construir estrategias para afrontar este nuevo escenario, ii) que el estudiante EMTP al momento del ingreso no posee los capitales cultural-académicos y sociales necesarios para enfrentarse exitosamente a la universidad y iii) que la institución universitaria requiere implementar cambios para responder a las necesidades de los estudiantes egresados de EMTP y primera generación universitaria.
\end{abstract}

Palabras clave: transición a la universidad, capital cultural, educación superior

\section{ABSTRACT}

This study explores the transition experience to the university of Professional Technical High School (PTHS) graduates who enter to Programa de Bachillerato, Universidad de Chile. The process of transition to the university of 6 freshmen students of Bachillerato in 2017 was investigated through semi-structured interviews. The results show: i) that admission to the university means a Rupture in the Trajectory of the student. From the possibility of entering the university, he must redefine his life project and build strategies to face the new scenario ii) that, at admission, PTHS student does not have the cultural-academic and social capital needed to successfully face university and iii) that the university institution needs to implement changes to meet the needs of students graduating from PTHS and first university generation.

Key words: transition to university, cultural capital, higher education

El financiamiento de la investigación proviene de los Fondos de Incentivo a la Investigación en Docencia de Pregrado de la Universidad de Chile y del Programa Académico de Bachillerato. 
Estudios Pedagógicos XLIV, $N^{\circ}$ 3: 271-288, 2018

LA UNIVERSIDAD COMO RUPTURA EN LA TRAYECTORIA EDUCATIVA: EXPERIENCIAS DE TRANSICIÓN DE ESTUDIANTES EGRESADOS DE ENSEÑANZA MEDIA TÉCNICO PROFESIONAL QUE INGRESAN AL PROGRAMA ...

\section{INTRODUCCIÓN}

En las últimas décadas, la Educación Superior ha vivido un proceso de masificación que ha tenido entre sus características la diversificación en la composición social de su matrícula, produciéndose la inclusión de sectores hasta entonces excluidos de ella (Puga, Atria, Fernández \& Araneda, 2017). Dicha expansión se sustenta en la creencia de que la Educación Superior sería el medio por excelencia para lograr la movilidad social (Sepúlveda, 2013), lo que genera un incremento en la aspiración por ingresar a ésta, especialmente en los sectores previamente excluidos de ella (Sepúlveda \& Valdebenito, 2014). Esto se comprueba en las cifras del Centro de Investigación y Desarrollo de la Educación (2003) que expresan las altas expectativas de ingreso a la Educación Superior y el fuerte incremento entre 2000 y 2003 de estas expectativas por parte de los estudiantes que cursan la Educación Escolar, lo que además se complementa con su alta disposición a endeudarse por ello (CIDE, 2012).

En este contexto, se comienza a identificar un nuevo sujeto universitario, caracterizado por ser la primera generación de su familia en ingresar a la Educación Superior. Esta situación tendrá un impacto profundo en su experiencia como estudiante dado que estos jóvenes no poseen acercamientos, ni en su vida cotidiana, ni en su historia familiar y social, a la universidad, de manera que ésta resulta una experiencia completamente nueva para ellos (Canales \& De los Ríos, 2009).

Sumado a lo anterior, existen cambios en las trayectorias de estos estudiantes, ya que anteriormente quienes no pertenecían a las capas que accedían a la Educación Superior vivían un rápido paso desde la niñez a la adultez, trabajando desde temprana edad, sin estudios o con muy pocos (Dávila \& Ghiardo, 2005). Esto se modifica a partir de la posibilidad de ingresar a la Educación Superior, cambiando esta trayectoria y redefiniendo las posibilidades de proyecto de futuro de estos sectores. En tal sentido, el paso a la universidad es un ámbito de la construcción de proyecto de vida, una "dimensión del curso de vida fuertemente anclada entre el pasado y el futuro inmediato, a la vez que un eje rector del presente y futuro cercano" (Puga et al., 2017: 149).

Por ende, una experiencia de transición exitosa del estudiante implica reconocer y abordar la gran distancia entre éstos y el mundo universitario, en términos sociales y culturales. En dicha experiencia de transición, la tarea de las instituciones de Educación Superior que se definen como transmisoras de conocimiento, juega un papel central. Actualmente, sin embargo, estas delegan la responsabilidad en el estudiante, como un asunto de esfuerzo individual (Canales \& De los Ríos, 2009).

La Universidad de Chile no ha estado ajena a dicha realidad. Sólo en 2017, un 46\% de los estudiantes que ingresaron fueron primera generación, superando al $45 \%$ que no lo es (no se tiene información del 9\% restante) (Universidad de Chile, 2017). Esto ha sido el resultado tanto del proceso de masificación descrito, como de la implementación progresiva de Políticas de Ingreso Alternativo a la universidad que abren cupos supernumerarios. Dichas políticas se impulsan tanto externa, como internamente de la institución. A nivel estatal, existen en la actualidad la Beca de Excelencia Académica (BEA) y el Programa de Acompañamiento y Acceso Efectivo a la Educación Superior (PACE). Algunas iniciativas propias de la Universidad de Chile son el Sistema de Ingreso Prioritario de Equidad Educativa (SIPEE) y el Programa Escuela de Desarrollo de Talentos (EDT), perteneciente a la Facultad de Economía y Negocios. Estas políticas buscan igualar las oportunidades de 
LA UNIVERSIDAD COMO RUPTURA EN LA TRAYECTORIA EDUCATIVA: EXPERIENCIAS DE TRANSICIÓN DE ESTUDIANTES EGRESADOS DE ENSEÑANZA MEDIA TÉCNICO PROFESIONAL QUE INGRESAN AL PROGRAMA ...

acceso a la Educación Superior, considerando la actual desigualdad en la calidad educativa que segmenta según sector socioeconómico.

El Programa Académico de Bachillerato de la Universidad de Chile (PAB), lugar desde donde se realiza la presente investigación, no ha estado exento de este contexto. Este es un programa académico de cuatro semestres de duración que conduce al grado académico de Bachiller con mención en ciencias naturales y exactas o en humanidades y ciencias sociales. Tras la obtención del grado, los estudiantes pueden transferirse a cualquiera de las carreras de la Universidad de Chile para completar sus estudios superiores a través de un mecanismo establecido para ello. Como en el resto de la Universidad de Chile, el PAB es un programa altamente selectivo al que ingresan estudiantes con altos puntajes.

El PAB ha sido testigo del cambio de escenario de la Educación Superior. En particular, del contexto de diversificación en la composición de la matrícula, expresado en el ingreso de mayor cantidad de estudiantes por vías especiales y que provienen de Establecimientos de Enseñanza Media con promedio de PSU inferior a 500 puntos (MINEDUC, 2009) y del aumento en los estudiantes egresados de programas formativos orientados al mundo del trabajo. Por ello y sumado a la alta exigencia del Programa, Bachillerato ha impulsado desde hace algunos años acciones de seguimiento y nivelación con el objeto de atenuar la brecha en las condiciones académicas iniciales de los estudiantes que así lo requieran. Por ejemplo, desde el año 2014, el PAB implementa un curso extensivo de la asignatura Matemáticas 1 (primer año) orientado a la nivelación de estudiantes que ingresan con menores puntajes PSU Matemática. El diseño de este curso implica más horas lectivas y mayor coordinación en la planificación y seguimiento de las estrategias de docencia, manteniendo los mismos resultados de aprendizaje esperados del curso regular (Cifuentes \& Mella, 2017). Esta experiencia ha dado resultados positivos, expresados en la mejora del rendimiento académico de sus participantes (Cifuentes, Munizaga 6 Mella, 2017).

Las acciones de seguimiento y nivelación se encuentran en muchos casos con obstáculos en diferentes ámbitos. En las universidades, la docencia se realiza como una actividad donde la responsabilidad del aprendizaje se deposita de manera total en el estudiante y donde la sala de clases obvia las desigualdades educativas y sociales de origen entre estos mismos. Así también, a nivel de las instituciones educativas y gubernamentales, las políticas se centran la mayoría de las veces en los apoyos económicos, sin asumir en toda su dimensión las necesidades de este nuevo tipo de estudiante.

Lo anterior da cuenta de la importancia de indagar y conocer la experiencia de los estudiantes de primera generación que ingresan a la universidad, en tanto reconocimiento de su existencia y de sus condiciones iniciales, entendido ello como un primer paso para trazar acciones institucionales orientadas a lograr una transición exitosa de éstos a la universidad.

Entre los estudiantes que son primera generación, uno de reciente aparición son aquellos que cursaron Enseñanza Media Técnico Profesional (EMTP). Su presencia en la Educación Superior ha aumentado porque existe una alta valoración de ésta, lo que se conjuga con las nuevas políticas de ingreso alternativo a la universidad que promueven su ingreso. El año 2017 un 5\% de los estudiantes que ingresaron a la Universidad de Chile provenían de EMTP (Universidad de Chile, 2017). En el PAB, los estudiantes provenientes de EMTP ingresan a través de vías regulares (PSU), alternativas (PACE, BEA y SIPEE) y a través del propedéutico de la Facultad de Economía y Negocios. Este consiste en un programa formativo que prepara durante $3^{\circ}$ y $4^{\circ}$ medio a estudiantes provenientes de 
EMTP para que ingresen a la Universidad de Chile. Bachillerato dispone vacantes para la totalidad de los estudiantes de dicho programa y en los últimos años ha ingresado al PAB aproximadamente un $10 \%$ de ellos, mientras el $90 \%$ lo hace a la Facultad de Economía y Negocios o a otras carreras.

El estudiante egresado de EMTP se constituye como un "caso ícono" de los estudiantes de primera generación. No es sólo habitualmente el primero de su familia en acceder a la universidad, sino que además proviene de un tipo de educación que tiene una orientación programática diferente a la universitaria. Mientras la EMTP tiene como finalidad la formación para el trabajo, la Universidad y Bachillerato de la Universidad de Chile se centran en la formación académica. Para el estudiante de EMTP, hasta hace muy poco tiempo acceder a la universidad era un deseo sin posibilidades reales de realización. Por lo mismo, el ingreso reciente de estos a la Educación Superior los encuentra con escasos recursos para enfrentarse a las exigencias de la vida universitaria.

El Programa Académico de Bachillerato busca generar un espacio de aprendizaje para todos los estudiantes, reconociendo, por ende, las transformaciones del estudiante que ingresa a ella. Por poseer un mayor desconocimiento del campo universitario, el estudiante de primera generación es un foco de prioridad. El PAB busca desarrollar en lo académico acciones orientadas a lograr un proceso de transición que les permita integrar en su vida la experiencia universitaria como una amplificación de recursos de carácter primeramente cultural, pero también social, que después retribuirán a la sociedad como profesionales. En este marco, este estudio se plantea indagar en la subjetividad de los estudiantes egresados de EMTP en su proceso de transición a la universidad.

\section{MARCO TEÓRICO}

\subsection{LA UNIVERSIDAD COMO ESPACIO SOCIAL EN DISPUTA}

El enfoque teórico desde el cual se comprenderá el espacio universitario es el de Pierre Bourdieu y Jean Claude Passeron (2009) que conceptualizan la educación considerando como elemento central la clase social, en tanto la Universidad es concebida por el autor como una institución estructurada para la permanencia de las clases altas, generando la exclusión de las clases bajas. Dicha permanencia de las clases altas estaría asegurada por la posesión de capitales económicos, sociales, culturales y simbólicos necesarios para 'generar distinción' y mantenerse en el campo universitario, capitales que no poseen y a los cuales no pueden acceder las clases bajas: "En una población estudiantil, no se accede más que al resultado final de un conjunto de influencias que se vinculan con el origen social y cuya acción se ejerce durante mucho tiempo" (Bourdieu \& Passeron, 2009: 29). En el caso de los estudiantes que aún con todos estos obstáculos logran ingresar a la universidad, el pasado social se transforma en déficit educativo, no existe roce previo con este campo, por lo que su tránsito se vuelve errático.

El espacio educativo puede comprenderse, desde la perspectiva de Bourdieu y Passeron (2009), como un campo social y como un juego. Como campo, al igual que un campo físico se encuentra compuesto por fuerzas en pugna, pero en este caso de carácter social; y como un juego, en cuanto existen ciertas reglas bajo las cuales se participa y los actores trazan estrategias para asegurar su posición en el campo, o para cambiar de posición (Bourdieu 

ESTUDIANTES EGRESADOS DE ENSEÑANZA MEDIA TÉCNICO PROFESIONAL QUE INGRESAN AL PROGRAMA ...

\& Passeron, 2009). La teorización de Bourdieu, a pesar de ser concebida y un producto de los estudios de la universidad francesa, encaja bastante bien con la realidad actual de la Educación Superior chilena, pues a partir de las reformas neoliberales en este ámbito, esta se ha transformado en un bien de consumo y es promovida para lograr la movilidad social, aunque las posibilidades de generar dicha movilidad estén cada vez más en entredicho, dado que el hecho de acceder a este nivel educativo no garantiza una trayectoria exitosa (Fukushi, 2013).

\section{LA UNIVERSIDAD COMO TRANSICIÓN A LA VIDA ADULTA Y LA TRAYECTORIA COMO CONSTRUCCIÓN ESTRATÉGICA Y VITAL}

La población que cursa estudios en la universidad es joven. El estudiante vive la experiencia universitaria al tiempo que el proceso de transición de la niñez a la adultez, que implica la definición y construcción de su proyecto de vida. Dávila, Ghiardo y Medrano (2005), conceptualizan este proceso de transición como un proceso lleno de cambios, con hitos que van marcando diferentes estructuras de transición. Las estructuras de transición son, en tal sentido, de carácter histórico y social: "La edad y las formas de hacerse adulto presentan variaciones que dependen de lo que cultural y socialmente se define para cada clase de edad y para cada sexo en cada clase de edad" (Dávila et al., 2005: 70).

De tal forma, se puede distinguir estructuras de transición tanto histórica como socialmente. Históricamente, porque los cambios sociales van generando estructuras diferentes y si nos remitimos a la actualidad encontraremos que la variable educación nunca había sido tan definitoria como hoy para dicha transición; y socialmente, porque las diferencias sociales producen a su vez estructuras diferentes. En este último caso podemos hablar del proceso de transición como un mecanismo de reproducción social, dado que lo que se reproduce es un proyecto de vida específico que ubica a los sujetos en una posición del espacio social, trazando con ello, lo que Dávila et al. (2005) denominan Trayectoria.

La Trayectoria es una construcción social y personal resultante del cruce entre la posición de los sujetos en el plano social y las disposiciones sociales producidas por estos en dicha condición social. Es decir, a cómo la clase social y los capitales asociados a ella se engarzan con ciertas representaciones, aspiraciones, expectativas y miradas de la vida que van dando paso a la adultez. Es en esta tensión donde se va construyendo una Trayectoria: "en ese juego, entre presente y futuro, entre sueños y decisiones, entre lo ideal y lo posible, los jóvenes se van haciendo adultos y ocupando un lugar en la sociedad, configurando su transición y trazando una trayectoria" (Dávila et al., 2005: 72). Comprenderemos así la trayectoria como el producto de la conjugación de: los objetivos fijados en el sujeto a partir de representaciones, aspiraciones, expectativas y miradas de vida; de las estrategias para llevarlos a cabo; y de los recursos o capitales acumulados para concretizar sus estrategias.

Respecto de su Trayectoria, las clases populares, medias y altas pasan cada vez más por las mismas etapas de transición, pero se distinguen en los tiempos que asignan a cada una de estas. Prácticamente todos pasan hoy por una estructura determinada por la secuencia niñez-estudios-trabajo, producto de la escolarización y masificación de la Educación Superior, aunque los tiempos de estudio aún son diferentes para cada clase. La estructura de transición en las clases populares es diferente en cuanto a los tiempos de duración de cada etapa. Los jóvenes de sectores populares estudian menos años y entran a trabajar a 
edades más tempranas que los de clase alta (Dávila et al., 2005). Lo que se mantiene es la segmentación del sistema de educación superior, pues las universidades más selectivas, lejos de ser más plurales, mantienen estructuras de reproducción de las elites (Brunner \& Uribe, 2007). Esta estructura se quiebra para el caso de estudiantes que egresan de EMTP e ingresan a Universidades de elite como la Universidad de Chile.

Los jóvenes populares que logran ingresar al campo universitario, particularmente a una institución de elite como la Universidad de Chile, constituyen un caso de Quiebre de Trayectoria. Es decir, si su trayectoria estaba direccionada hacia el ingreso temprano al mercado laboral facilitado por estratégicamente por cursar EMTP, la posibilidad de ingresar a la Educación Superior se abrirá como una nueva estrategia para mejorar su posición futura. En este quiebre de la Trayectoria se abre un horizonte para la construcción de nuevas disposiciones subjetivas y para el cambio de posición objetiva. Nos referiremos, por tanto, al "Quiebre de Trayectoria" como una ruptura que desencadena un proceso de tensión objetiva y subjetiva, en clave de Bourdieu (2009) de lucha por la apropiación de nuevos capitales, a partir de la generación de nuevas aspiraciones y expectativas en el espacio social universitario. Este quiebre de trayectoria, como lugar que reúne procesos subjetivos del joven estudiante y condiciones objetivas del campo educativo y de la clase social de origen, desencadena un proceso conflictivo de lo que se tiene y lo que se necesita para mantenerse y tener éxito en los estudios universitarios. Significa por ende, que manteniéndose el objetivo de mejorar la posición, lo que cambia son las estrategias desplegadas para ello (ingreso a la universidad), asociada a una serie de recursos que el estudiante no habría acumulado hasta ese entonces.

\section{MÉTODO}

Para lograr el objetivo de indagar en la experiencia de transición de los estudiantes egresados de EMTP a la Universidad de Chile, específicamente al PAB, se realizó un estudio de carácter exploratorio-descriptivo. Se definió utilizar la metodología cualitativa, pues esta se orienta al conocimiento de la subjetividad humana, buscando entender los fenómenos sociales desde la perspectiva del actor, como fuerza motora de la acción (Taylor y Bogdan, 1987), cuestión que resulta central en la investigación, en tanto los jóvenes construyen disposiciones y valoraciones que son parte de una identidad y constituyen proyectos que se remueven a partir del ingreso a la universidad.

\subsection{INSTRUMENTO Y PROCEDIMIENTO DE PRODUCCIÓN DE DATOS}

El instrumento de producción de información escogido fue la entrevista, ya que su flexibilidad y apertura permite una mayor profundidad en la indagación de la subjetividad del estudiante. Las entrevistas fueron realizadas entre septiembre y noviembre de 2017. Su diseño fue semiestructurado y contempló las áreas que se muestran en la tabla a continuación. 
LA UNIVERSIDAD COMO RUPTURA EN LA TRAYECTORIA EDUCATIVA: EXPERIENCIAS DE TRANSICIÓN DE ESTUDIANTES EGRESADOS DE ENSEÑANZA MEDIA TÉCNICO PROFESIONAL QUE INGRESAN AL PROGRAMA ...

Tabla 1. Pauta guía de entrevista

\begin{tabular}{|l|l|}
\hline \multicolumn{1}{|c|}{ Temas a cubrir } & \multicolumn{1}{c|}{ Aspectos en los que indagar } \\
\hline $\begin{array}{l}\text { Motivaciones para ingresar a } \\
\text { la Universidad de Chile }\end{array}$ & $\begin{array}{l}\text { Cómo llegó al Bachillerato } \\
\text { Motivaciones principales de ingreso a la universidad } \\
\text { Percepción de los recursos al ingresar a la universidad }\end{array}$ \\
\hline Experiencia en la universidad & $\begin{array}{l}\text { Cumplimiento de expectativas } \\
\text { Dificultades académicas } \\
\text { Relación con los compañeros } \\
\text { Relación con los profesores }\end{array}$ \\
\hline Expectativas futuras & $\begin{array}{l}\text { Intención de seguir estudiando en la universidad y en la carrera de } \\
\text { preferencia al momento de ingresar al PAB } \\
\text { Aspectos que considera más importantes para mantenerse en el Bachillerato }\end{array}$ \\
\hline
\end{tabular}

La entrevista se estructuró como relato ordenado temporalmente desde el estudio en EMTP en adelante. Se buscaba ahondar con ello en aspectos objetivos, como son los capitales que poseen y las características institucionales de Bachillerato; y subjetivos, como la experiencia, representaciones y expectativas de la universidad con respecto a sus objetivos y proyecto futuro.

\subsection{MUESTRA}

La muestra estuvo conformada por estudiantes egresados de EMTP, usando como criterios en su diseño: el sexo, la participación en el propedéutico de la Facultad de Economía y Negocios (Escuela de Desarrollo de Talentos, EDT) y el año de egreso de la Educación Secundaria. Se definió como "estudiantes de trayectoria temprana" a quienes ingresan a la Educación Superior el año inmediatamente posterior al de egreso de Enseñanza Media, y como "estudiantes de trayectoria tardía" a los estudiantes que no ingresaron a la Educación Superior el año inmediatamente posterior al egreso de Enseñanza Media, sino que lo hicieron posteriormente.

Para seleccionar los estudiantes que compondrían la muestra se contaba con acceso a la base de datos de estudiantes del Bachillerato. Se contactó a los estudiantes en persona, ya que existe un vínculo institucional que permitía un acceso más directo a éstos. La muestra quedó conformada como se muestra en la tabla a continuación:

Tabla 2. Composición de la muestra

\begin{tabular}{|c|c|c|c|}
\hline Sujeto $\mathbf{N}^{\circ}$ & Tipo de Trayectoria & Participación en EDT & Sexo \\
\hline 1 & Temprana & Sí & Masculino \\
\hline 2 & Temprana & Sí & Femenino \\
\hline 3 & Temprana & No & Femenino \\
\hline 4 & Temprana & No & Femenino \\
\hline 5 & Tardía & No & Masculino \\
\hline 6 & Tardía & No & Masculino \\
\hline
\end{tabular}




\subsection{ANÁLISIS DE LA INFORMACIÓN}

Las entrevistas tuvieron una duración promedio de una hora. Para el análisis, se utilizó una codificación abierta, la cual se fue contrastando y haciendo dialogar con el marco teórico. Se inició con algunos conceptos centrales intuitivos y se incluyeron y modificaron categorías durante el análisis.

La técnica de análisis usada fue el Análisis de Contenido (Andréu, 2000). Se trabajó a partir de la discusión continua de cada una de las categorías por parte del equipo investigador, a través de una primera revisión general para esbozar un mapa de categorías y luego una segunda revisión y discusión para afinar las categorías.

\section{RESULTADOS}

Los resultados se estructuran en tres momentos relevantes: i) el ingreso a la universidad como un quiebre con la trayectoria previa, ii) la transición a la universidad como un choque con un nuevo campo institucional y iii) la proyección futura como respuesta al quiebre de trayectoria no resuelto. Estos momentos se sintetizan en la figura $\mathrm{N}^{\circ} 1$. Se añade además un cuarto punto referido a la construcción y exploración vocacional de los estudiantes en Bachillerato y un quinto punto referido a las diferencias entre los estudiantes de trayectorias tempranas y tardías.

Figura 1. Síntesis del proceso de transición del estudiante a la universidad

\section{Quiebre de Trayectoria}

A partir de la posibilidad de ingreso a la universidad, el estudiante accede a una nueva estrategia para mejorar su posición social. Sin embargo, no posee los recursos necesarios para sustentarla en términos de capital culturalacadémico: no existe ni acercamiento previo a la universidad desde la familia (primera generación), ni un nivel académico adecuado (formación orientada al trabajo).

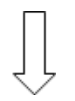

\section{Choque con el carácter de la institucionalidad en la adquisición de recursos}

La institucionalidad universitaria, a pesar de su alta exigencia, ofrece una docencia excluyente para este tipo de estudiante, pues no contempla su nivel inicial y está excesivamente centrada en el contenido, sin incorporar estrategias de enseñanza. El estudiante tiene mal rendimiento académico, se frustra y no logra explorar su vocación.

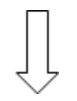

\section{Quiebre no resuelto}

El estudiante egresado de EMTP no logra incorporar los recursos necesarios para enfrentarse a la experiencia universitaria, por lo que el Quiebre de Trayectoria no queda resuelto. Sin embargo, el haber ingresado a la universidad una vez genera en él persistencia, pues una vez que ha ingresado no desiste del deseo de estudiar una carrera universitaria. Lo que cambiará será la táctica para lograrlo, buscando alternativas como rendir la Prueba de Selección Universitaria para ingresar directamente a la carrera deseada. Sin embargo, esto no resuelve su transición a la universidad, en la medida que no ha adquirido los recursos cultural-académicos para afrontarla. 


\subsection{INGRESO A LA UNIVERSIDAD: EL QUIEBRE DE TRAYECTORIA}

Hablamos de un quiebre de Trayectoria en el momento de ingreso del estudiante a la universidad porque, dentro del camino que recorre, los recursos acumulados hasta ese entonces no se corresponden con los que necesita para enfrentarse al tránsito universitario. Las estrategias que se han ido construyendo a lo largo de la vida de estos jóvenes, influidas en gran medida por la familia y su historia, se relacionan al ingreso pronto al mercado de trabajo y no con el ingreso a la universidad. El recurso utilizado en la estrategia de clase es la obtención de un título técnico de nivel inicial: "Me metí un técnico porque uno no sabe lo que va a pasar, entonces dije, si pasa alguna cosa que no tenga en mente, por lo menos tener algo con qué afrontar la vida" (Mujer, trayectoria temprana).

Las estrategias que resultan más realistas, en dicho contexto, son las asociadas a conseguir cualificación para ingresar al mercado del trabajo con una mejor posición, razón por la cual estos jóvenes ingresan, motivados generalmente por sus familias, a la Educación Media Técnico Profesional. No obstante, la aspiración de ingresar a la universidad se encuentra ya presente, como deseo de mejorar su vida. Esta aspiración se concreta de forma imprevista al presentárseles la posibilidad de ingresar a la Educación Superior. Ésta se ve, por ende, como oportunidad y no como una estrategia construida por estos: "Es que vi la oportunidad, o sea, me estaban dando la oportunidad de enseñarme, de prepararme, entonces como dicen 'ah, la U te da un título más grande, para ganar mucho dinero', entonces por eso lo decidí” (Hombre, trayectoria temprana, EDT).

Esta posibilidad ha sido además abierta porque son los estudiantes de mejor nivel en sus establecimientos educativos de procedencia:

Mi colegio está asociado con la UTEM, entonces cuando tú entras al colegio te dicen que el diez por ciento de mejor nivel entra gratuitamente a la universidad. En ese momento no había gratuidad, entonces como que las universidades son lejanas para todos y como en ese momento, con mis buenas notas, se abrió la posibilidad de entrar a la universidad (Mujer, trayectoria temprana).

A pesar de que los jóvenes logran ingresar por su buen rendimiento académico, lo cual resultaría meritorio, a nivel subjetivo el ingreso a la Educación Superior se traduce para el joven estudiante como una presión o una carga familiar y de clase, por ser el único que lo logró:

No. Siempre quise entrar a la universidad y mi idea no era tomarme un año, porque muchas veces se pierde el hilo o después [...] la universidad, el trabajo, y también que nadie de mi familia había entrado a la universidad, entonces era una carga que me hacía yo misma [...] la primera, de todos, porque todos los demás, varios como que han estudiado algo, pero no en una universidad (Mujer, trayectoria temprana).

Lo que significa ingresar a la universidad es mucho más que la adquisición de herramientas técnicas, más bien es salir de una posición 'inferior' en la escala social, para arribar a un futuro mejor que las generaciones precedentes:

Mi motivación principal es cumplir lo que quiero, y también, por ejemplo, mi papá que siempre me dice: tú tienes que ser más que yo. Él es mecánico, se quedó ahí por 
Estudios Pedagógicos XLIV, N $^{\circ}$ 3: 271-288, 2018

LA UNIVERSIDAD COMO RUPTURA EN LA TRAYECTORIA EDUCATIVA: EXPERIENCIAS DE TRANSICIÓN DE ESTUDIANTES EGRESADOS DE ENSEÑANZA MEDIA TÉCNICO PROFESIONAL QUE INGRESAN AL PROGRAMA ...

dejado. Mi papá era técnico, mi mamá también, pero mi papá se quedó ahí, nunca ejerció. Sí, porque dice que su trabajo era sucio, que le cuesta ganarse la plata (Mujer, trayectoria temprana).

Es decir, el ingreso a la universidad representa una posibilidad para salir de la posición actual y aspirar a una mejor. Por ende, la elección de la universidad, más que estar guiada por motivos académicos, usa como criterio central la capacidad de mejorar la posición social:

[Mi papá] es mecánico en mantención en empresas. Y él me dice que no me vaya a la USACH también por eso, porque él dice que como él conoce el manejo de la empresa, dice que siempre uno de la Católica o de la Chile, eh, es el jefe, el de la USACH como que puede ser jefe, pero siempre va a ser mandado y él me dijo que no entrara a la USACH (Mujer, trayectoria temprana, EDT).

Al aparecer de forma imprevista en el proyecto del estudiante la posibilidad de concretizar el ingreso a la Universidad, las condiciones del estudiante, construidas por su trayectoria previa, chocan con los recursos existentes para dar el giro. Hasta antes del quiebre, los jóvenes EMTP han desarrollado recursos asociados a mejorar su posición social, usando la educación técnica como herramienta para el ingreso temprano al mercado laboral. Es recién en el momento en que ingresan a la universidad cuando los jóvenes comienzan a vislumbrar que deberán construir otro conjunto de recursos, asociados a una trayectoria de otras características. En tal sentido, se trata de un quiebre, porque los recursos acumulados en la trayectoria previa al ingreso a la universidad no se corresponden con los recursos para entrar en la "lucha" del campo universitario. En otras palabras, ni las orientaciones subjetivas, ni los capitales acumulados de forma estratégica hasta ese entonces son un recurso para jugar en el nuevo campo.

En cuanto a los recursos para afrontar este quiebre de trayectoria, los estudiantes identifican que ellos mismos se encuentran en desventaja respecto de las herramientas académicas para responder a las exigencias universitarias, es decir, poseen un bajo capital cultural-académico al momento de ingresar:

Yo tuve muy buen rendimiento académico en el colegio, pero yo nunca estudié, yo era de las que se sacaba 6.5 o 7.0 y no había estudiado nada. Y aquí entras a Bachi o yo creo que a cualquier carrera es lo mismo, pero tienes que estudiar y enfocarte un poco en eso, y yo ya sabía que se me iba a hacer difícil porque no tenía un proceso, un método de estudio, pero como que cada vez se hace más imposible (Mujer, trayectoria temprana).

La estructuración de los programas de EMTP orientadas al mundo del trabajo pone en una posición de inicio desventajosa a los estudiantes que egresan de este tipo de educación, lo que sumado a la falta de hábito de estudio hace muy difícil revertir esta posición:

[...] pero es difícil, porque no tenía muchos conocimientos sobre biología, por ejemplo, sociología y eran materias que no me interesaban, entonces al ser materias que no me interesan aún, es más difícil seguir el ritmo de los demás, porque los demás también 
tienen preparación en eso, en cambio uno no, uno pasó los ramos de especialidad y en primero y segundo algo de Filosofía o de Física, pero nada más (Hombre, trayectoria temprana, EDT).

Los estudiantes atribuyen a este desnivel inicial lo difícil que resulta para ellos la transición a la universidad: "Yo creo que es el desnivel no más, el desnivel es harto, porque vengo de colegio público, igual no venía con el hábito de estudiar ni nada de eso, entonces eso igual me ha costado" (Hombre, trayectoria temprana, EDT).

Sumado a ello existe una carencia de redes sociales para afrontar este campo. El entorno en el cual el estudiante se desenvuelve no ingresa a la universidad, por lo que difícilmente pueda traspasar alguna noción sobre lo que esta es, o sobre las formas de enfrentarla. El imaginario de la universidad se encuentra construido, por ende, sobre supuestos lejanos o ideas muy generales de ésta:

Es que mis papás nunca entraron, nunca hicieron una carrera universitaria, el mundo... o sea, me vine acá, llegué a la Chile por así decirlo solo, porque ninguno de mi liceo tampoco vino a la Chile, entonces con quién me desenvolvía era solamente con la gente de acá, respecto al tema (Hombre, trayectoria tardía).

\subsection{LA TRANSICIÓN: EL CHOQUE CON LA INSTITUCIONALIDAD UNIVERSITARIA Y LA FALTA DE RECURSOS PARA AFRONTAR EL NUEVO CAMINO}

En el proceso de transición a la universidad los estudiantes viven de forma violenta el choque con su dinámica de funcionamiento y las exigencias académicas instaladas. Sus primeros resultados en las evaluaciones hacen patente la distancia entre los recursos que estos poseen y los del estudiante que tradicionalmente ingresa a la universidad. En este proceso, su identidad se ve afectada y la frustración es alta, pues provienen de un campo en el cual ellos se sienten adelantados, el de la educación secundaria a la cual ingresaron y se insertan en un nuevo campo donde viven con impotencia que no poseen los recursos para afrontarlo.

Al estudiante, el proceso lo hace reflexionar y darse cuenta de esta distancia, sin embargo, la corta duración del proceso formativo y la alta exigencia de Bachillerato limita una salida positiva al proceso, puesto que el estudiante toma consciencia del proceso que está viviendo y va construyendo estrategias para afrontarlo cuando ya es tarde para enrumbar camino:

Primero, al principio como que echaba la culpa que nunca me pude afirmar en las materias y todo eso, después empecé a pensar que tal vez como siempre se me dio la facilidad, siempre me fue fácil estudiar y entrar en un lugar donde sí o sí tenía que sentarme en un escritorio y estudiar, estudiar, estudiar, cosa que yo no tenía esa rutina, me cambió, o sea, no supe cómo hacer el tránsito en ese cambio, entonces ahí empecé a reflexionar que ya, que quizás vine con una base mala, pero también tenía que poner de mi parte, pero esa reflexión fue ya después que me había echado los ramos...(Hombre, trayectoria tardía).

Aquí es donde entran a jugar los aspectos institucionales dado que, lejos de facilitarlo, obstaculizan aún más el proceso de transición de éste tipo de estudiante. Para los estudiantes 
egresados de EMTP, el camino se va haciendo cada vez más cuesta arriba, puesto que el formato institucional cada vez les recalca con más fuerza sus carencias de capitales. Las bajas notas en el primer ciclo de evaluaciones del año les hacen muy difícil mejorar en el segundo y, dado los mecanismos de transferencia a las carreras, sienten que partir con malas notas prácticamente les cierra la posibilidad de ingresar a las carreras que desean.

En cuanto a la construcción de recursos académicos para afrontar Bachillerato, la institucionalidad en el ámbito de la docencia se encuentra adaptada al estudiante tradicional, tanto en su nivel académico de inicio, como en el formato de enseñanza. En cuanto al nivel académico de ingreso, no se reconocen las diferencias entre los estudiantes de trayectorias diferentes:

Me costó mucho acostumbrarme, igual pasé Matemáticas, no me fue tan mal, pero era como, los contenidos que nosotros veíamos en el colegio a los contenidos que vi en la universidad eran totalmente distintos, porque al menos yo en tercero y cuarto medio, que fue cuando tuve técnico, teníamos una hora y media de clases a la semana, entonces no, no veíamos nada. Y en la universidad como que dicen 'no, si eso lo vieron en tercero medio', y es como 'no, yo no lo vi' (Mujer, trayectoria temprana).

Sobre la docencia propiamente tal, el profesor no es un recurso movilizador del aprendizaje, dejando esta tarea totalmente puesta en el estudiante:

Y después igual yo cuando estaba en Química yo hablé con el profesor, de hecho hablé con todos los profesores del área científica, o sea, Química, Biología y ahora Física, porque yo le dije al profe que venía de técnico, cómo podía estudiar para poder pasar el ramo y el profe me dijo que tenía que pedir el Chang y estudiar sola (Mujer, trayectoria temprana, EDT).

Los estudiantes sienten que existe una idea de la docencia basada en competencia y en mantener en la universidad a "los mejores". Dicha docencia no movilizaría el aprendizaje, sino que responsabiliza a los estudiantes de su logro. En el imaginario de los estudiantes, los profesores no se centran en la enseñanza, sino en la selección:

Acá hay... no sé, hay mecanismos como que... como que... eh, como que manchan ese... la forma de enseñar, por ejemplo, eso mismo del sistema de filtros para los cupos, cosas así, o esa misma idea que tienen algunos docentes de mantener alto el nivel, 'así que empecemos a pasar materia así el primer año bien difícil o bien rápido y que queden los mejorcitos no más y después empezamos a trabajar con ellos', esa lógica igual creo yo que es mala... o sea, mala para mí, porque tal vez para los académicos sea buena, mantienen el nivel, no los juzgo (Hombre, trayectoria tardía).

Sólo para el caso de la asignatura que ha sido intervenida para responder a las necesidades de los estudiantes con menores recursos académicos, como ocurre con Matemática extensiva, se reconoce la existencia de una docencia adaptada al estudiante:

[...] en Matemáticas G, no, tienen la noción de partir desde cero por así decirlo, de hecho al principio partimos de nociones así como súper básicas, muy obvias, pero que 
LA UNIVERSIDAD COMO RUPTURA EN LA TRAYECTORIA EDUCATIVA: EXPERIENCIAS DE TRANSICIÓN DE ESTUDIANTES EGRESADOS DE ENSEÑANZA MEDIA TÉCNICO PROFESIONAL QUE INGRESAN AL PROGRAMA ...

de repente ponían ejemplos de que esas cosas obvias no eran tan obvias, entonces... formas distintas de enseñar (Hombre, trayectoria tardía).

Esto se expresa en una formación orientada a la comprensión de los fundamentos de la matemática:

[...] para empezar los profesores de Matemática $\mathrm{G}$ son los mejores profesores que hay al menos en el área matemática, porque no sé, a mis compañeros de Matemática regular como que les pasan la materia tan rápido, que no les explican, como que sólo les dicen "ya, esto se hace y así se aprende", en cambio nuestros profesores nos dicen 'ya, esto se hace así por estas razones', y como que nos demuestran por qué se hace así (Mujer, trayectoria temprana).

Sin embargo, dicha asignatura constituye una excepción en la docencia de Bachillerato. En general esta se caracteriza por estar diseñada para el estudiante tradicional de la universidad, aquel que no tenía un desnivel de base. En términos subjetivos, esta estructura es frustrante para el estudiante, puesto que todo su esfuerzo no se reconoce en lo logrado en términos de obtención del cupo para las carreras que estos quieren transferirse:

Aparte que uno pasa ramos y nadie te asegura que vas a tener cupo, yo creo que eso debe ser, es lo peor, porque te esfuerzas, te esfuerzas y tienes... pasas todos tus ramos y tienes, no sé, un promedio 4,9, pero tu 4,9 no sirve para el cupo, porque el cupo corta en $5[\ldots]$ (Mujer, trayectoria temprana).

Existe, en resumen, una estructura de Bachillerato caracterizada por una corta duración (cuatro semestres) y alta exigencia, una docencia no diseñada para el nuevo estudiante y mecanismos de transferencia a las carreras asociados a su éxito académico, que chocan con las condiciones de los estudiantes provenientes de EMTP y dificultan una transición exitosa.

\subsection{EL QUIEBRE NO RESUELTO: MANTENCIÓN DE ESTRATEGIA Y REDEFINICIÓN DE LA TÁCTICA}

La vivencia de este proceso genera en los estudiantes una reevaluación de su permanencia en la universidad, tanto de la carrera a estudiar como de los medios para ingresar a ésta:

Siempre, desde el primer momento que entré a Bachi dije 'voy a dar la PSU a final de año', entonces cuando yo vi que ya me había echado un ramo, entonces había perdido prioridad para Plan Común, que era Beauchef, entonces empecé a considerar la idea de salirme de Bachillerato para preparar la PSU (Mujer, trayectoria temprana, EDT).

La incapacidad de construir en un corto tiempo los recursos para enfrentarse a la exigencia de Bachillerato, cuestión difícil para un estudiante que proviene de un EMTP, sin base en al menos tres de los ramos de primer año (Biología, Química y Física), tiene como resultado un rendimiento deficiente para ingresar a las carreras que estos se plantean. La alternativa pasa a ser rendir la PSU a final de año e intentar ingresar a las carreras por esta vía: 
Estudios Pedagógicos XLIV, $\mathrm{N}^{\circ}$ 3: 271-288, 2018

LA UNIVERSIDAD COMO RUPTURA EN LA TRAYECTORIA EDUCATIVA: EXPERIENCIAS DE TRANSICIÓN DE ESTUDIANTES EGRESADOS DE ENSEÑANZA MEDIA TÉCNICO PROFESIONAL QUE INGRESAN AL PROGRAMA ...

Sí, reprobé dos ramos y me dijeron que máximo tenía que aprobar, o sea, mínimo, ocho ramos. Entonces como ya me eché dos, este semestre tengo que sí o sí aprobarlos todos, y por eso también evalué la posibilidad de irme, dar la PSU este año por si acaso (Mujer, trayectoria temprana).

\subsection{LA VOCACIÓN IRRESUELTA}

Un aspecto que se logra observar en el discurso de los estudiantes es que el proceso de elección de la carrera que estudiarán, luego de cursar Bachillerato, no es de exploración vocacional, sino el producto de una constante adaptación de sus expectativas a sus condiciones reales. Bachillerato, en ese sentido, les abre un campo nuevo a disciplinas desconocidas por ellos, pero al mismo tiempo los recursos con que cuentan y la estructura del mismo, le cierran la posibilidad de estudiar algunas carreras, generalmente las más deseadas socialmente y que les asegurará una mejor posición social futura.

Esto se traduce en que la opción por la carrera se ajusta a "lo conocido" en términos de experiencia vivida por los estudiantes y recursos acumulados, buscando carreras coherentes con su trayectoria académica previa, dando cuenta que opera una racionalidad siempre adaptada a la experiencia ya construida. Los estudiantes que provienen del propedéutico de la Facultad de Economía y Negocios (EDT), se inclinarán por las carreras de esta facultad; en el caso de quienes no pasaron por éste, la elección de la carrera a estudiar es resultado de un proceso de descarte que se va efectuando a través de la experiencia en Bachillerato.

Entrevistadora: ¿Y por qué [Ingeniería] Comercial? ¿Cómo llegaste a esa decisión? Entrevistado: Bueno, en realidad no estudiaría ahí en Comercial, pero era porque como estudié Administración dije 'ah, las dos cosas se pueden complementar y me puede ser más fácil', pero mi opción favorita era todavía lo de los computadores, las redes y esas cosas (Hombre, trayectoria temprana, EDT).

Bachillerato es para los estudiantes EMTP el primer lugar para acercarse a la universidad. Sin embargo, esta experiencia inicial es insuficiente para resolver la vocación del estudiante, sobre todo porque la necesidad de responder a la alta exigencia académica genera un proceso confuso de exploración vocacional, donde no queda claro cuáles son las razones por las que se abren y cierran opciones para los estudiantes:

Es que yo en el colegio saqué el título de Asistente Contable, así que estudiar Contabilidad era como la opción que mi mamá veía como mucho más viable porque ya sabía eso. Pero a mí no me gusta, entonces busqué como otras opciones, entre esas, no sé, Administración Pública me gustó demasiado, pero como que ahora en Bachi me di cuenta que no, las Humanidades no son lo mío, así que igual como que sirvió de algo (Mujer, trayectoria temprana).

\subsection{DIFERENCIAS ENTRE LOS ESTUDIANTES DE TRAYECTORIAS TEMPRANAS Y TARDÍAS}

Los estudiantes de trayectoria temprana y trayectoria tardía tienen algunas diferencias constatadas en la investigación, las que sólo podemos enunciar en este artículo como tesis a comprobar a futuro, por el poco nivel de profundidad con que aparecen en las entrevista. 
LA UNIVERSIDAD COMO RUPTURA EN LA TRAYECTORIA EDUCATIVA: EXPERIENCIAS DE TRANSICIÓN DE ESTUDIANTES EGRESADOS DE ENSEÑANZA MEDIA TÉCNICO PROFESIONAL QUE INGRESAN AL PROGRAMA ...

El estudiante de trayectoria temprana tiene menos tiempo de acumulación de recursos para ingresar a la universidad. El espacio temporal que han tenido para procesar sus aspiraciones, ajustar sus expectativas con respecto a sus logros y comprender qué necesitan para sortear la universidad es menor. Acumulan una gran ansiedad por el logro y viven con gran frustración e intensidad cada paso que dan. El ingreso a la universidad de estos estudiantes es siempre mediante cupo de ingreso especial, con escasa planificación ya que es el resultado de que aprovecharon una oportunidad que aparece como irrepetible, lo que genera una gran presión social por no decepcionar a sus familias, por lograr lo que nadie más ha logrado en su entorno.

El estudiante de trayectoria tardía, a diferencia de lo anterior, ha vivido un camino más largo para ingresar a la universidad. Ya sea porque se preparó un año más a través de preuniversitario, o porque exploró otras posibilidades de futuro laboral durante el o los años posteriores a su egreso de EMTP; el tiempo que ha pasado y la experiencia vivida le hacen planear con más variables y más conocimiento su transición a la universidad. Esto genera que sean más asertivos con respecto a sus necesidades, que la frustración sea menor dado que han procesado sus expectativas previamente, o porque durante el tiempo de intervalo han podido acumular algún tipo de capital específico para afrontar la universidad. Los estudiantes de trayectoria tardía, más que aprovechar las oportunidades ofrecidas en el caso de los cupos especiales, han creado su propia oportunidad a través de la generación de recursos propios.

\section{DISCUSIÓN Y CONCLUSIONES}

El Quiebre de Trayectoria aparece como concepto definitorio para comprender la transición del estudiante a la Universidad de Chile. El impacto subjetivo de dicho quiebre define el carácter de la transición, en tanto los objetivos y estrategias asociados al proyecto de vida de los jóvenes que son primera generación en la universidad chocan con los nuevos objetivos y con las estrategias que están por construirse para enfrentarlas, al cumplirse la aspiración de ingreso a la universidad.

El hecho de que exista un quiebre en la transición entre educación secundaria y universidad se debe a la segmentación de clase propia de la sociedad chilena, que en su expresión educativa construye futuros diferentes para clases diferentes. Quienes estudian en EMTP se proyectan hacia un futuro laboral inmediato o a la continuidad de estudios técnicos, mientras quienes estudian en Enseñanza Media Científico-Humanista en establecimientos de mejor calidad se proyectan al ingreso a universidades. Por ende, la Educación Superior se constituye como espacio de consolidación de la posición o de mejora de esta. Quienes ingresan a partir de vías de acceso alternativo o especial a la Educación Superior representan, una "excepción a la regla", a quienes se les abre como posibilidad lo que aspiran para concretar la movilidad social.

La universidad aparece, para los estudiantes, como oportunidad que sería irracional desaprovechar, sin embargo, las referencias y nociones de lo que esta es y cómo enfrentarla están escasamente construidas en el medio social, siendo muy vagas: se tiene claridad de que la Universidad de Chile proveerá de una "mejor posición social” pero no de los recursos académicos necesarios para enfrentarse a ella. La posición de entrada del estudiante es con más dudas que certezas, pero a la vez tensiona la identidad del estudiante, que pasa de estar entre 
los mejores estudiantes de su generación en la educación secundaria a sentirse con recursos insuficientes para enfrentarse al nuevo medio, lo que genera frustración a nivel subjetivo.

Las principales diferencias entre el estudiante tradicional de la Universidad de Chile y aquel que egresa de EMTP son en términos de recursos para afrontar el camino universitario en dos ámbitos: capital cultural-académico y capital social. El capital cultural académico es escaso dado que los estudiantes egresados de EMTP tienen un déficit de contenidos por los programas propios de su rama de estudios, y una carencia de métodos o estrategias de estudio, particularmente porque no poseen la práctica de estudiar largas horas para aprender una determinada materia.

La escasez de capital social se expresa en que el mundo social que rodea al estudiante no tiene experiencia universitaria, por lo cual poseen un imaginario escaso de los recursos a construir en la universidad y nadie con experiencia que les pueda aconsejar o traspasar conocimiento sobre cómo moverse en dicho ámbito.

Las características institucionales con las que el estudiante se encuentra pueden permitir abordar de forma exitosa el quiebre de trayectoria, lográndose la transmisión de capital cultural-académico suficiente para la transición y con los ajustes de la estructura curricular necesarios para emprender un nuevo camino con éxito o, por el contrario, pueden profundizar la carencia de recursos para enfrentarse a esta. En este caso, el estudio devela que la Universidad de Chile no está adaptada a las condiciones del estudiante que es primera generación en la universidad.

Lo anterior se afirma en que la docencia, mayoritariamente, se ejerce como espacio de reproducción de contenidos, adaptada al estudiante que tradicionalmente ingresa a la Educación Superior, el cual posee mayores condiciones para movilizar su propio proceso de aprendizaje. Y segundo, porque la alta exigencia en el corto periodo de duración de Bachillerato choca con los tiempos que requiere un estudiante para nivelarse, dificultando lograr una transición exitosa a la universidad. El resultado de dicho choque con la institucionalidad es la reevaluación de las tácticas para la transición a la universidad, como es rendir nuevamente la PSU. Esto invita a la institución a replantearse cómo hacer frente a este choque.

A partir de estas conclusiones sobre la actual realidad institucional y sus capacidades para abordar a los estudiantes que viven el quiebre de trayectoria, es necesario que la institución se repiense, considerando que estudiantes que provienen de otras trayectorias educativas toman un mayor tiempo en vivir este tránsito y en construir los recursos para enfrentar el campo universitario. En tal sentido, tiempos más largos le permitirían una mejor acumulación de capitales para enfrentar la experiencia educativa.

Por otra parte, los resultados interpelan a los procesos nacionales de selección e ingreso a la universidad y la falta de acompañamiento de estos. En efecto, los actuales procesos de selección de los estudiantes intervienen poco o nada en los recursos que requiere el estudiante para abordar el proceso educativo, enfocados netamente en la definición de criterios sociales y académicos para seleccionar a los estudiantes y poco en el proceso de acompañamiento en la universidad. Los programas de ingreso a la Educación Superior a nivel nacional, como son los Cupos de Equidad, la Beca de Excelencia Académica y el Programa de Acompañamiento y Acceso Efectivo a la Educación Superior (PACE) se centran en seleccionar al estudiante mediante criterios de vulnerabilidad y en que cumpla el puntaje mínimo PSU requerido por la institución a la cual ingresará, pero no en que este posea las herramientas académicas para hacer una transición exitosa a la universidad. 
LA UNIVERSIDAD COMO RUPTURA EN LA TRAYECTORIA EDUCATIVA: EXPERIENCIAS DE TRANSICIÓN DE ESTUDIANTES EGRESADOS DE ENSEÑANZA MEDIA TÉCNICO PROFESIONAL QUE INGRESAN AL PROGRAMA ...

En síntesis, el indagar en las experiencias de transición a la universidad de los estudiantes egresados de EMTP permite mostrar la necesidad de levantar acciones institucionales orientadas a un acompañamiento efectivo e integral del estudiante para transitar exitosamente en la nueva trayectoria.

Entre ellas, referido al déficit de capital cultural-académico, la creación de estructuras que contemplen tiempos más largos y se orienten a la nivelación, que permitan al estudiante egresado de EMTP y a los estudiantes que son primera generación en la universidad incorporar de forma efectiva el capital cultural-académico que la institución tiene para transmitir.

\section{REFERENCIAS BIBLIOGRÁFICAS}

Andréu, J. (2000). Las técnicas de análisis de contenido: Una revisión actualizada. Fundación Centro Estudios Andaluces, 10(2), 1-34.

Bourdieu, P. \& Passeron, J. (2009). Los herederos. Los estudiantes y la cultura. Buenos Aires: Siglo XXI editores.

Brunner, J. \& Uribe, D. (2007). Mercados universitarios: el nuevo escenario de la educación superior. Santiago: Ediciones Universidad Diego Portales.

Canales, A. \& De los Ríos, D. (2009). Retención de estudiantes universitarios vulnerables. Calidad en la educación, 30, 50-83.

Centro de Investigación y Desarrollo de la Educación. (2003). IV Encuesta Nacional a los Actores del Sistema Educativo. Santiago: CIDE. Recuperado el 2 de noviembre desde http://biblioteca. uahurtado.cl/ujah/reduc/pdf/pdf//mfn248.pdf

Centro de Investigación y Desarrollo de la Educación. (2012). IX Encuesta a Actores del Sistema Educativo. Santiago: CIDE. Recuperado el 2 de noviembre desde http://www.cide.cl/ documentos/Informe_IX_Encuesta_CIDE_2012.pdf

Cifuentes, M. \& Mella, J. (2017). Separar para integrar: Una experiencia de nivelación en matemáticas en el Programa Académico de Bachillerato de la Universidad de Chile. En Ó. Jerez y S. Colleen (Eds.), Innovando en Educación Superior: Experiencias clave en Latinoamérica y el Caribe 2016-2017 (pp. 133-142). Santiago: Facultad de Economía y Negocios, Universidad de Chile.

Cifuentes, M., Munizaga, F. \& Mella, J. (2017). Más tiempo para aprender: Evidencias para aportar al debate sobre equidad, inclusión y gratuidad de la Educación Superior a partir de resultados de dispositivos de nivelación matemática. Pensamiento Educativo. Revista de Investigción Educacional Latinoamericana, 54(1), 1-15. doi: 10.7764/PEL.54.1.2017.1

Dávila, Ó. \& Ghiardo, F. (2005). Trayectorias, transiciones y condiciones juveniles en Chile. Nueva Sociedad, 200, 114-126.

Dávila, Ó., Ghiardo, F. y Medrano, C. (2005). Los Desheredados. Trayectorias de vida y nuevas condiciones juveniles. Valparaíso: Ediciones CIDPA.

Fukushi, K. (2013). Una aproximación cualitativa al estudiante de primera generación en la educación superior chilena. En Aequalis (Ed.), Acceso y permanencia en la educación superior: Sin apoyo no hay oportunidad (pp. 115-144). Santiago: Aequalis.

MINEDUC. (2009). Lineamientos estratégicos para la discusión de una política de mediano plazo para la educación media. Apartado 1: Diagnóstico de la educación media. Recuperado el 9 de noviembre desde https://es.scribd.com/document/82099129/Lineamientos-Educacion-MediaDiagnostico\#

Puga, I., Atria, R., Fernández, R. \& Araneda, C. (2017). Proyectos de ida y oportunidades en la educación media. Nuevas demandas sociales al sistema escola chileno. Última década, 47, 118153. 
Estudios Pedagógicos XLIV, N $^{\circ}$ 3: 271-288, 2018

LA UNIVERSIDAD COMO RUPTURA EN LA TRAYECTORIA EDUCATIVA: EXPERIENCIAS DE TRANSICIÓN DE

ESTUDIANTES EGRESADOS DE ENSEÑANZA MEDIA TÉCNICO PROFESIONAL QUE INGRESAN AL PROGRAMA ...

Sepúlveda, L. (2013). Más jóvenes en la educación superior, ¿mayores esperanzas de igualdad social?: una reflexión sobre las aspiraciones de futuro de los estudiantes secundarios chilenos. Cuadernos de Educación, 51, 1-9.

Sepúlveda, L. \& Valdebenito, M. (2014). ¿Las cosas claras? Aspiraciones de futuro y proyecto educativo laboral de jóvenes estudiantes secundarios. Estudios Pedagógicos, 40(1), 243-261.

Taylor, S. \& Bogdan, R. (1987). Introducción a los métodos cualitativos de investigación. Buenos Aires: Paidós.

Universidad de Chile. (2017). Informe de admisión y caracterización de estudiantes nuevos 2017. Santiago. Recuperado el 9 de noviembre desde http://repositorio.uchile.cl/handle/2250/143757 